\title{
A LOCALIZAÇÃO DOS ATORES DO SISTEMA DE INOVAÇÃO BRASILEIRO E SEUS IMPACTOS REGIONAIS NA DÉCADA DE 2000
}

\author{
Ulisses Pereira dos Santos \\ CEDEPLAR/FACE/UFMG \\ Philipe Scherrer Mendes \\ CEDEPLAR/FACE/UFMG
}

\begin{abstract}
Resumo: A literatura sobre sistemas de inovação e desenvolvimento regional observa a existência de impactos positivos da proximidade geográfica de atividades econômicas e estruturas de ciência e tecnologia. Frente a esta observação, o presente trabalho avalia se a localização de atores do sistema brasileiro de inovação favorece as atividades econômicas locais em microrregiões brasileiras. Os resultados de testes econométricos indicam que o setor industrial das microrregiões observadas é positivamente influenciado pela presença de entes do sistema de inovação. Isso sugere que o processo recente de expansão do sistema de inovação no Brasil tende a gerar importantes efeitos sobre as estruturas produtivas regionais, principalmente, sobre a indústria. Para o setor serviços não foram observados impactos estatisticamente significantes, embora os dados indiquem que a presença de tais atores também favorece o setor.
\end{abstract}

Palavras Chave: Sistemas de Inovação, Desenvolvimento Regional, Dados em painel.

\section{THE LOCATION OF THE ACTORS OF BRAZILIAN INNOVATION SYSTEM AND ITS REGIONAL IMPACTS IN THE 2000s}

\begin{abstract}
The literature on innovation systems and regional development points the existence of positive impacts of geographical proximity of scientific and technological structures over economic activities. Based on this observation this paper aims to evaluate the effects of the localization of Brazilian innovation system actors over local economic activities. The econometric results show that industry sector is positively affected by the presence of those actors in the same region. This results suggest that the late expansion in Brazilian innovation system may have important effects over regional productive structures, mainly over industry. For the tertiary sector statistically significant impacts were not found. Nevertheless, the data suggest that the regional presence of the innovation system actors favors the sector.
\end{abstract}

Key words: Innovation Systems, Regional Development, Panel Data.

Área ABEIN: Área 4 - Redes e sistemas urbanos, regionais e nacionais

4.3 Sistemas de inovação - nacional, regional, setorial, tecnológico

Classificação JEL: R11, O30 


\section{A LOCALIZAÇÃO DOS ATORES DO SISTEMA DE INOVAÇÃO BRASILEIRO E SEUS IMPACTOS REGIONAIS}

\section{Introdução}

O Brasil passou, ao longo da década de 2000, por um processo de expansão de seu Sistema Nacional de Inovação, que resultou na ampliação de sua abrangência territorial. Desta forma, não apenas aumentou o número de instituições de ensino e pesquisa e as produções científica e tecnológica, como estas também passaram por um processo de redistribuição espacial, contemplando um conjunto ampliado de localidades perante ao território nacional.

A partir da literatura acerca das relações entre os sistemas de inovação e o desenvolvimento regional, acredita-se que tal processo possa ser benéfico para a dinâmica dos setores produtivos, dado que a proximidade geográfica em relação a uma estrutura de ciência e tecnologia geraria spillovers positivos sobre as atividades econômicas da região (AUDRETSCH; FELDMAN, 1996; COOKE, 1998; JAFFE, 1989). À luz desta perspectiva, o presente artigo tem como objetivo avaliar a existência de impactos da localização de atores do Sistema Nacional de Inovação sobre a dinâmica das atividades econômicas regionais no Brasil. Em linhas gerais, tenta-se verificar se a existência numa dada região de tais atores é, de fato, benéfica para as atividades econômicas nela localizadas. Para isso é adotado aqui um indicador capaz de sumarizar informações relativas ao grau de desenvolvimento das estruturas microrregionais do Sistema Nacional de Inovação, o IDRSI, proposto inicialmente por Santos (2014). Esse indicador é confrontado com dados relativos à dinâmica econômica dos setores indústria e serviços para as 558 microrregiões brasileiras a partir de estimações econométricas. Além do grau de desenvolvimento das estruturas locais de ciência e tecnologia, são utilizadas variáveis de controle com vistas a avaliar determinantes da dinâmica dos setores, incluindo variáveis relativas aos possíveis efeitos do desempenho de localidades vizinhas sobre a indústria e os serviços das microrregiões analisadas.

Busca-se com esse trabalho, portanto, preencher uma lacuna na literatura acerca dos sistemas de inovação e o desenvolvimento regional no Brasil, de modo a observar como a localização de estruturas de ciência e tecnologia pode influenciar a dinâmica econômica das regiões no país. Trata-se de um aspecto altamente relevante dado o processo recente de ampliação no número de universidades e centros de pesquisa no Brasil, com a sua interiorização.

A sequência deste artigo é composta por cinco seções, além desta introdução. A segunda seção apresenta aspectos teóricos sobre os sistemas de inovação e o desenvolvimento regional. A terceira seção aborda a evolução do sistema brasileiro de inovação entre os anos de 2000 e 2010 . A quarta e a quinta seções tratam da análise econométrica realizada para este artigo, sendo apresentados, respectivamente, aspectos relativos à metodologia e os resultados dos exercícios empíricos. A última seção apresenta as considerações finais do trabalho.

\section{Sistemas de inovação e desenvolvimento regional}

O sistema nacional de inovação (SNI) é formado por um conjunto de instituições formais e informais que promovem os processos de inovação tecnológica, sendo o grau de interação entre elas determinado pelo ambiente em que estão inseridas (JOHNSON, 1995). Trata-se de uma rede composta por instituições pertencentes às esferas pública e privada que atuam de modo a criar, importar, modificar e difundir novas tecnologias por meio de suas atividades e interações (FREEMAN, 1987). O SNI é composto, portanto, 
pelos atores econômicos, sociais, políticos, organizacionais, e institucionais que têm relevância para a difusão e uso das inovações tecnológicas (EDQUIST, 2005).

Os objetivos principais de um sistema de inovação são: i) a realização de pesquisa e desenvolvimento; ii) a construção de competências internas por meio de qualificação e treinamento; iii) o suporte às interações necessárias para o processo inovativo e iv) a criação, ou mudança, de instituições eliminando obstáculos e dando suporte à inovação, à incubação e ao financiamento de atividades de inovação, dentre outros (EDQUIST, 2005). Com isso, é possível mencionar dentre os componentes do SNI atores como as universidades, institutos de pesquisa públicos e privados, centros de $\mathrm{P} \& \mathrm{D}$ empresariais e agências governamentais, além das empresas.

A definição do conceito de SNI parte do entendimento que um sistema é constituído não apenas por um determinado conjunto de agentes, mas também pelo relacionamento entre estes, o que determina seu grau de desenvolvimento e suas potencialidades (LUNDVALL, 1995). A inovação tecnológica é, desta forma, identificada como um processo social que é impulsionado pela existência deste conjunto de agentes e instituições, que, uma vez alinhados às trajetórias tecnológicas vigentes, interagem com vistas a facilitar o seu desenvolvimento. Estes agentes integrantes de um determinado sistema de inovação se caracterizariam pela participação num processo conjunto de aprendizado onde a constante troca de informações originaria um mecanismo de causação circular, pelo qual cada um destes influenciaria os demais (LUNDVALL, 1995). Trata-se, portanto, de um processo que pressupõe a existência de externalidades partindo de determinados elementos do SNI e atuando sobre os outros, acabando por influenciar o desenvolvimento de um processo de inovação.

Nesse sentido, pode-se considerar de extrema importância a capacidade de comunicação e interação entre todos os agentes constituintes do SNI. Tanto as instituições voltadas para atividades relacionadas à ciência e à tecnologia quanto aquelas que compõem o setor produtivo devem se esforçar na sustentação de fluxos informacionais, para que seja assegurado o sucesso do processo de inovação em todas as suas facetas. Dado isto, num sistema de inovação bem desenvolvido, as empresas e os agentes que as ajudam a promover a inovação devem interagir estabelecendo tais fluxos de informação, sustentando o devido alinhamento entre o conhecimento científico e o conhecimento técnico.

Acredita-se, por isso, que aspectos espaciais, territoriais e regionais guardam importante relação com o processo de inovação tecnológica, especialmente no que tange a estes fluxos informacionais entre os diversos agentes que participam dos processos de produção científica e tecnológica (ASHEIM; GERTLER, 2005; COOKE, 1998, 2001; JAFFE, 1989). A proximidade geográfica, associada à partilha de elementos sociais e culturais, facilitaria e reforçaria tais fluxos de modo a impulsionar a inovação nos setores produtivos. Nesse sentido, além das organizações, das firmas, das interações e das relações de aprendizado para a atividade inovativa, apontadas por autores como Freeman (1987, 1995), Lundval (1995), Nelson e Rosenberg (1993) como os principais determinantes da atividade de inovação, deve-se considerar também a importância de elementos regionais para esse processo (COOKE, 1998).

Dentre os neoschumpeterianos responsáveis por introduzir o conceito de sistema de inovação, Freeman $(1987$; 2002) foi o primeiro a apontar a importância de políticas regionais sobre os processos de catch up. Ao tratar das políticas de desenvolvimento econômico no Japão, o autor ressaltou o esforço governamental na criação e execução de políticas regionais baseadas na ciência, educação, comunicações e infraestrutura. $O$ objetivo de tais políticas seria levar capacitações técnicas a todo o território nacional de modo a integrar aos circuitos tecnológicos e científicos o maior número possível de 
agentes, independente de sua localização ou escala econômica (FREEMAN, 1987, p. 36).

Acerca das regiões subnacionais, o autor observa que a aglomeração das atividades industriais teria importantes repercussões sobre o seu desenvolvimento, em razão das economias de aglomeração marshallianas (FREEMAN, 2002). Desta forma, remete a aspectos amplamente difundidos no âmbito da economia regional ao chamar a atenção para a tendência das atividades econômicas em se aglomerar no espaço, com foco, sobretudo, na existência de um contingente de trabalhadores qualificados e regionalmente identificados e no fortalecimento dos fluxos de informação.

No âmbito da economia regional, o conceito de Sistemas Regionais de Inovação (SRI) foi introduzido por Cooke (1992), partindo da influência neoschumpeteriana. Tal conceito deve ser entendido como uma síntese dos desenvolvimentos acerca da relação entre inovação tecnológica e o desenvolvimento regional e os SNIs. Deve-se considerar que a busca por estratégias para o desenvolvimento regional por meio a atividade inovativa originou uma série de estudos, teorias e políticas entre as décadas de 1980 e 1990. Dentre os conceitos oriundos deste período, e que contribuíram para o desenvolvimento de um conceito de SRIs, é possível mencionar os de complexos tecnológicos regionais, parques tecnológicos, redes tecnológicas, tecnopolos, milieu inovativo, entre vários outros. Todos estes relacionavam a ascensão da inovação tecnológica a aspectos regionais (COOKE, 1998). Na medida em que tais conceitos, explorados predominantemente por cientistas regionais, foram atrelados aos esforços teóricos acerca dos sistemas nacionais de inovação, baseados principalmente em Freeman (1987; 1995) e Lundvall (1995), ganhou forma o conceito de sistemas regionais de inovação (COOKE, 1998). Trata-se, então, de um conceito inspirado na ideia de SNIs, mas com ênfase em sistemas de inovação regionalmente delimitados (ASHEIM; GERTLER, 2005).

Assim como os sistemas nacionais de inovação, os sistemas regionais estão baseados na existência de um corpo institucional e nas interações entre os agentes que o compõem. Porém, esta perspectiva agrega à análise aspectos mais comuns à economia regional, principalmente aqueles atrelados aos efeitos da proximidade entre os agentes no estabelecimento de suas relações. Podem ser mencionados, nesse sentido, fatores ligados à imersão regional dos agentes num mesmo cenário local como facilitadores das interações necessárias para o bom andamento do processo inovativo (COOKE, 2001).

O conceito de SRIs pondera que a geografia não é incidental, mas sim fundamental para a ascensão das inovações, e assume a distribuição desigual do conhecimento no espaço (ASHEIM; GERTLER, 2005). Nesse sentido, as diferenças em termos da capacidade inovativa das regiões seriam resultantes da existência de diferentes trajetórias de aprendizagem regional imersas em sistemas institucionais diferenciados (ISAKSEN, 2001). Portanto, a compreensão das especificidades regionais marcadas pela proximidade espacial dos agentes e a sua interação na condução da inovação, de fato, importariam para o devido entendimento dos fatores que a favorecem. O SRI pode ser definido como a infraestrutura institucional para o suporte da atividade de inovação em meio à estrutura produtiva de uma determinada região (ASHEIM; GERTLER, 2005). A base estrutural para um sistema regional de inovação consistiria, então, na aglomeração de um conjunto de firmas, apresentando interações entre si, em centros e institutos de pesquisa e ensino superior e de pós-graduação e em laboratórios privados de P\&D. Também integrariam este sistema as agências de transferência de tecnologia, associações comerciais e as agências e departamentos governamentais relevantes para a atividade de inovação e para o desenvolvimento econômico (COOKE, 1998). Todos estes atores teriam suas interações e ações destinadas à promoção da inovação pautadas 
pelas políticas governamentais neste sentido. Dado isto, pode-se dizer que a estruturação de um SRI estaria atrelada à existência de quatro conjuntos de elementos fundamentais. Seriam eles, as firmas, as instituições públicas e privadas, a infraestrutura de conhecimento e a política de inovação (DOLOREUX, 2002). Nesse cenário, o conjunto de firmas compondo uma aglomeração produtiva regionalmente identificada, o conjunto de instituições voltadas para a produção e difusão do conhecimento científicotecnológico, e as relações interativas entre estas duas esferas seriam os principais determinantes do dinamismo e desenvolvimento para um SRI (ISAKSEN, 2001). A viabilização destas interações seria o principal foco das instituições e políticas voltadas para a promoção da inovação.

Uma vez estabelecidos e disseminados os conceitos de SNI e SRI, ascende uma questão relativa às possíveis relações entre eles. Ou seja, faz-se necessário considerar se os sistemas nacionais e subnacionais de inovação apresentam características concorrentes ou complementares. Quanto a isso, Freeman (2002), motivado pelas mudanças na economia mundial, tratou da importância de se identificar e interpretar as relações entre os sistemas continental, nacional e subnacional de inovação. $O$ autor ressalta o ganho de importância das escalas subnacionais nos estudos acerca da inovação, apontando a necessidade de entendimento das relações de complementariedade e concorrência entre estas distintas escalas para as novas possibilidades de desenvolvimento tecnológico e econômico. Freeman (2002) chama a atenção, então, ao fato de que as vantagens regionais oriundas da aglomeração seriam influenciáveis por fatores ligados à escala nacional. Fatores como como a cultura, política, economia e instituições tecnológicas nacionais teriam importante influência sobre tais benefícios regionais dos quais as atividades territorialmente aglomeradas se aproveitariam. Logo, tais aspectos de natureza nacional poderiam limitar ou ampliar os benefícios da aglomeração regional. Por isso, Freeman (2002) identifica uma relação de complementaridade entre os sistemas nacional e subnacional de inovação.

Edquist (2005) também trata sobre as relações entre escalas e limites geográficos em termos dos sistemas de inovação. Segundo este autor, a importância dos limites geográficos para os sistemas de inovação é definida tanto por fatores nacionais quanto regionais. Nesse sentido, afirma que para países de grandes dimensões, propensos à maior diversidade entre as regiões que os compõem, o emprego do conceito de sistemas regionais de inovação - SRIs - seria mais relevante que o de sistemas nacionais de inovação - SNI. Por outro lado, pondera que os aspectos legais e políticos, que influenciariam tais SRIs, estariam em algumas circunstancias relacionados aos governos nacionais, mesmo nos países de grandes dimensões territoriais. Por isso, o emprego da perspectiva regional estrita ficaria comprometido. Com isso, o autor chega a uma conclusão próxima à de Freeman (2002), considerando que os sistemas de inovação em escalas nacional e subnacional teriam caráter mais complementar que excludente.

Com isso, assume-se aqui que a relação vigente entre os sistemas nacional e subnacional de inovação seria de complementariedade, estando o SRI sujeito às práticas e políticas exercidas na esfera nacional. Portanto, os SRIs são considerados parte integrante do SNI, assumindo-se que cada região pode apresentar uma trajetória tecnológica própria que pode determinar o sucesso ou fracasso das políticas nacionais de ciência e tecnologia. Em outros termos, assume-se que o SNI apresenta um caráter espacial e que esse caráter é determinante em seu desenvolvimento e no desenvolvimento regional no país. Considera-se, portanto, que estruturas locais de ciência e tecnologia originárias de políticas e esforços nacionais podem apresentar variações em seu nível de desenvolvimento, de acordo com a sua localização geográfica, o que estaria relacionado às diferentes trajetórias tecnológicas regionais e às 
bases de conhecimento locais (ASHEIM; GERTLER, 2005; OINAS; MALECKI, 1999). Aceita-se, portanto, que o ambiente econômico exerce influência sobre os agentes de origem nacional nele inseridos (COOKE, 1998; ISAKSEN, 2001).

De forma similar, acredita-se que uma região em que se localizem alguns dos principais atores do SNI tenderia a se desenvolver mais, já que estaria em vantagem na atração de novas indústrias, em razão de sua melhor estrutura de ciência e tecnologia. Isso, por sua vez, culminaria no crescimento da produção e da renda internas, assim como da sua arrecadação. A maior capacidade econômica destas regiões possibilitaria a elas o reinvestimento em sua infraestrutura regionalizada de aprendizagem $\mathrm{e}$ conhecimento, a qual poderia impulsionar o sistema regional de inovação em questão, ampliando sua a competitividade interna e externamente (ASHEIM, 1996; FLORIDA, 1995). Deste modo, tais regiões teriam maiores condições de alcançarem o desenvolvimento regional. Por sua vez, regiões que não contem com o mesmo investimento do governo nacional na instalação de atores do SNI em seus limites geográficos teriam menores possibilidades de promoção de inovações tecnológicas, o que limitaria sua competitividade e, consequentemente, suas possibilidades de desenvolvimento. Logo, a distribuição espacial dos entes do SNI teria implicações sobre a desigualdade de desenvolvimento entre as regiões nacionais.

Com isso, acredita-se que a análise regional do SNI seja fundamental para o entendimento das diferenças de desenvolvimento econômico entre as regiões subnacionais. Na sequência desse trabalho, assume-se que a distribuição espacial do SNI pode ser um fenômeno ligado ao grau de desenvolvimento econômico regional.

\section{Evolução recente do Sistema Brasileiro de Inovação}

O SNI brasileiro é considerado ainda imaturo no que tange ao seu nível de desenvolvimento, dado que apresenta indicadores relativos à produção científica e tecnológica muito inferiores aos observados para países já desenvolvidos (ALBUQUERQUE, 1999). No entanto, deve-se observar que o SNI brasileiro passou, no período entre os anos de 2000 e 2010, por um processo de expansão, sendo esta pautada, sobretudo, nos avanços do sistema de ensino e pesquisa. Nesse sentido, foi possível identificar crescimento em indicadores que se referem a esta faceta do SNI, como o número de artigos científicos publicados em periódicos de abrangência internacional e o número de docentes em cursos de pós-graduação (mestrado e doutorado) no país, como pode ser observado por meio da tabela 1. Tal avanço pode ser creditado ao forte aumento no número de instituições de ensino superior no país. Indicadores como o número de bolsas de estudos para formação no exterior também apresentaram expansão no decorrer da década. Desta forma, a esfera científica do SNI brasileiro avançou tendo como principal resultado desse processo o aumento na produção de artigos científicos publicados em periódicos internacionais.

Tabela 1

Evolução do SNI brasileiro: 2000 - 2010

\begin{tabular}{l|ccc}
\hline & $\mathbf{2 0 0 0}$ & $\mathbf{2 0 1 0}$ & Crescimento (\%) \\
\hline Patentes & 7.259 & 8.582 & 18,2 \\
Artigos científicos & 5.095 & 29.958 & 488,0 \\
Docentes de pós-graduação & 29.190 & 60.038 & 105,7 \\
Microrregiões com patentes & 253 & 285 & 12,6 \\
Microrregiões com artigos científicos & 175 & 355 & 102,9 \\
Microrregiões com cursos de pós-graduação & 68 & 115 & 69,1 \\
\hline
\end{tabular}

Fonte: Elaboração própria a partir de ISI, INPI e GeoCapes 
Todavia, é observável que a produção tecnológica, ilustrada pelo número de pedidos de patentes no período, não apresentou o mesmo vigor em seu crescimento que o verificado para as atividades científica e de formação profissional. É possível deduzir, a partir desse cenário, que o avanço no SNI brasileiro foi baseado nas esferas científica e de ensino, fortemente ligadas ao setor público no Brasil. Já a esfera tecnológica, que refletiria o esforço dos setores produtivos, apresentou um ritmo de crescimento menos empolgante. Esse aspecto seria relacionado à estrutura produtiva brasileira e ao baixo dinamismo da indústria doméstica, marcada pela ausência de interações com a esfera científica do SNI. Esse descompasso entre as esferas científica e tecnológica figura como um dos principais entraves ao desenvolvimento econômico brasileiro (RIBEIRO et al,.2010; SUZIGAN; ALBUQUERQUE, 2011).

Outro aspecto relevante, no que tange à tabela 1 , diz respeito ao ganho de abrangência regional do SNI brasileiro, entre os anos de 2000 e 2010. Observa-se que o número de microrregiões com registros de patentes, artigos científicos e docentes de pós-graduação aumentou ao longo do período. Nesse sentido, é possível considerar que o avanço do SNI brasileiro foi acompanhado de um processo de redistribuição espacial de seus atores, fazendo com que um número maior de localidades passasse a contar com a presença de seus entes. Novamente cabe destaque à produção científica, uma vez que para 2010 o número de localidades que apresentaram tal atividade foi o dobro do observado em 2000. O número de microrregiões com cursos de pós-graduação também apresentou considerável crescimento ao longo do período observado.

Tal expansão territorial do sistema de ensino e pesquisa pode ter reflexos importantes sobre o desenvolvimento regional no país. Primeiro, por permitir um avanço na oferta de trabalho qualificado ao longo do território nacional e, em segundo lugar, por dotar um conjunto maior de localidades de infraestrutura de pesquisa. Dessa forma, o desenvolvimento de pesquisas científicas identificadas com as realidades locais pode gerar efeitos positivos sobre as trajetórias tecnológicas regionais. Logo, são criadas condições para replicar em maior escalar os casos bem sucedidos de interação entre ciência e tecnologia (SANTOS; DINIZ, 2013; SUZIGAN; ALBUQUERQUE, 2011) ${ }^{1}$.

Por sua vez, a atividade tecnológica também apresentou menor ritmo de expansão territorial. Esse dado pode indicar que a expansão no sistema de ensino e na produção científica ainda não geraram os devidos transbordamentos sobre a atividade tecnológica ou que as esferas produtivas não reuniram, ainda, condições de absorver possíveis ganhos oriundos do aumento na produção científica brasileira. No entanto, deve-se levar em conta as limitações do número de depósitos de patentes como indicador proxy para a atividade tecnológica. Isso ocorre, pois, nem toda inovação tecnológica é, de fato, patenteável. Ou seja, há inovações em processos, organizacionais, de serviços e mesmo de produtos que não são passíveis de patenteamento. Ademais, o processo de registro de uma patente pode ser demorado e apresentar altos custos, criando uma defasagem temporal entre a inovação e seu patenteamento. Com base em tais aspectos, tem-se conhecimento que parte dos impactos do crescimento da estrutura de ensino e pesquisa no país pode não estar contemplado por dados de patentes, aqui utilizados. Ainda assim, trata-se do indicador com maior difusão na literatura orientada para o estudo do

\footnotetext{
1 Suzigan e Albuquerque (2011) observam casos de sucesso no que tange à interação universidadeempresa no Brasil, sendo os principais relativos ao setor mínero-metalúrgico, em Minas Gerais, e ao aeronáutico em São Paulo. A proximidade geográfica entre o sistema de ensino e o setor produtivo transparece como um elemento importante na análise dos autores. Santos e Diniz (2013) realizam análise similar, mas focada na interação universidade-empresa na siderurgia de Minas Gerais.
} 
desempenho tecnológico de países ou regiões, o que justifica sua adoção (ALBUQUERQUE, 1999; ALBUQUERQUE et al., 2002; GRILICHES, 1990).

Cabe considerar que mesmo com o processo recente de ampliação do SNI, o país em pouco avança no que tange à distância em relação aos SNIs de países mais desenvolvidos. Observar-se que o ritmo de crescimento das produções científica e tecnológica brasileiras necessita ser ampliado para que se possa, de fato, alcançar os países mais avançados econômica e tecnologicamente (RIBEIRO et al., 2010). Acredita-se que tal incremento no SNI brasileiro deve ser orientado pela continuidade de sua redistribuição territorial.

\section{Aspectos metodológicos}

\section{Base de dados}

A base de dados para a análise empírica foi construída na forma de um painel balanceado, contendo informações dos anos de 2000 e 2010 para todas as 558 microrregiões do Brasil, totalizando 1116 observações.

Para avaliar os efeitos dos atores regionais do sistema nacional de inovação sobre as atividades de serviços e o setor industrial foi utilizada como variável o Índice de Desenvolvimento Regional do Sistema de Inovação (IDRSI). Este índice foi desenvolvido por Santos (2014) com vistas a identificar as mudanças na distribuição regional dos entes do SNI brasileiro entre 2000 e 2010. Trata-se de um indicador obtido por meio do método de análise fatorial a partir de três variáveis que abrangem as atividades científica, tecnológica e de qualificação profissional no âmbito do SNI. Tais variáveis são o número de artigos científicos publicados por milhão de habitantes, o número de patentes por milhão de habitantes e o número de docentes de pós-graduação por milhão de habitantes para cada microrregião brasileira. O número de artigos científicos foi obtido a partir de pesquisa junto ao sitio do Institute of Science Information (ISI Web of Science), do qual foram identificados os artigos publicados por residentes no Brasil em periódicos indexados pela instituição para os anos de 2000 e 2010. O número de artigos científicos é recorrentemente utilizado como proxy para a produção científica pela literatura acerca dos sistemas de inovação (ALBUQUERQUE, 1999). O número de depósitos de patentes por microrregião é oriundo de uma tabulação especial fornecida pelo Instituto Nacional de Propriedade Industrial (INPI). Dados de patentes, por sua vez, configuram a principal proxy para a produção tecnológica (JAFFE, 1989). Por fim, o número de docentes de pós-graduação foi obtido perante consulta junto à base de dados do portal GeoCapes. Estes dados foram ponderados pelas populações microrregionais disponibilizadas pelo portal Ipeadata.

O modelo de análise fatorial, utilizado para a criação do IDRSI a partir das variáveis acima descritas, leva a uma redução da dimensionalidade conjunto original de variáveis num número menor de fatores que resumiriam suas informações originais (MINGOTI, $2005)^{2}$. Uma vez obtidos os fatores, é possível calcular seus valores numéricos para cada elemento amostral. Cada um desses valores $\mathrm{Fi}$, denominado escore do fator i, resume o conjunto de informações refletidas pelas variáveis originais de análise padronizadas, para cada observação i, conforme a equação abaixo.

$\mathrm{Fi}=\mathrm{c} 1 \mathrm{iZi}+\mathrm{c} 2 \mathrm{iZi}+\mathrm{c} 3 \mathrm{iZi}$

Os valores do IDRSI são obtidos, portanto, pelos escores F referentes a cada uma das microrregiões observadas. Nesse sentido, o índice proposto é uma combinação linear das variáveis acima apontas, a qual sumarizaria suas variáveis em um único indicador.

2 Mais detalhes sobre o modelo de análise fatorial podem ser obtidos em Mingoti (2005). 
O IDRSI, é definido, então, pelos escores do primeiro fator obtido ao se aplicar o método de análise fatorial para os períodos de 2000 e 2010 aos dados referentes ao SNI brasileiro em suas 558 microrregiões, como definido pela equação abaixo:

IDRSIi = c1i(Patentes/milhão de hab i $)+$ c2i(Artigos/milhão de habi $)+c 3 i($ Docentes de pós/milhão de habi)

Os coeficientes cji são os pesos para a ponderação das variáveis utilizadas na composição do IDRSI, sendo estimados pelo método dos mínimos quadrados ordinários, com base na matriz de loadings, que representam a correlação entre as variáveis originais e os fatores oriundos da análise fatorial. (MINGOTI, 2005). Disso, conclui-se que o IDRSI consiste numa soma ponderada dos valores das três variáveis originais utilizadas em sua construção, para cada microrregião, sendo os fatores de ponderação os coeficientes que expressam o relacionamento do fator com a variável a que se refere (SANTOS, 2014). Logo, quanto maior for o valor para o IDRSI, maior será a participação de atores do SNI na microrregião considerada. Cabe considerar que os scores para os fatores obtidos por análise fatorial são padronizados, de modo a apresentar média igual a 0 e desvio padrão igual a 1.

O principal propósito do trabalho é identificar como o IDRSI afeta o desempenho da atividade industrial e do setor de serviços em uma perspectiva regional, com vistas a avaliar os possíveis efeitos do processo de expansão territorial do SNI brasileiro sobre as atividades econômicas ao longo de suas microrregiões. Cabe destacar que entre 2000 e 2010 foi identificado num crescimento superior a $40 \%$ no total de microrregiões brasileiras com IDRSI superiores à média nacional, indicando uma maior abrangência territorial do SNI brasileiro. Destaca-se que para a análise proposta outras variáveis de controle foram utilizadas para o desenvolvimento do exercício empírico proposto.

Parte dos dados utilizados foi extraída do IBGE (PIB, PIB industrial e PIB - serviços). Os dados, municipais, foram agrupados por microrregião e ponderados pela população. Outra transformação feita nos dados foi a construção de variáveis de ponderação espacial. A ideia da construção destas variáveis está fundamentada na tentativa de tratamento econométrico para as possíveis influências territoriais, características do objeto de análise. Entende-se que ao avaliar os fatores que determinam o desempenho industrial e do setor de serviços é importante levar em consideração possíveis influências do entorno ${ }^{3}$. A variável de exportações foi obtida no AliceWeb/MDIC. O mesmo tratamento de agrupar em microrregiões, relativizar pela população e ponderar pela possível influência do entorno foi feita para esta variável. Importante destacar a inexistência de dados (municipalizados) de exportações para o ano de 2000. A alternativa adotada foi utilizar os dados de $2003^{4}$ como proxy.

\section{Metodologia}

O propósito do trabalho de identificar como a variável IDRSI determina o desempenho industrial e do setor de serviços esbarra na tentativa de encontrar uma especificação econométrica que minimize erros de omissão de variáveis explicativas e que permita o avanço nos tratamentos metodológicos necessários à identificação do modelo.

Partindo de uma primeira especificação de análise da causalidade de IDRSI em PIB (industrial) e PIB (serviços), tem-se:

$\mathrm{Y}_{\text {industrial }}=\alpha+\beta *$ IDRSI $+\varepsilon$

3 Este aspecto será melhor abordado na descrição da metodologia.

$4 \mathrm{O}$ ano de 2003 foi o primeiro que houve disponibilização de dados municipais de exportações. 
$\mathrm{Y}_{\text {serviços }}=\alpha+\beta *$ IDRSI $+\varepsilon$

Assume-se que nesta primeira especificação o modelo passa a ter problemas de viés de omissão de variável relevante, que seriam capturadas nos termos de erro e tornariam os $\widehat{\beta}$ não confiáveis.

Outras variáveis de controle foram incluídas nas estimações, na tentativa de avançar na identificação do melhor modelo. Para os determinantes do desempenho industrial são incluídas variáveis de exportações (per capta), dummy para o ano de 2010. Para os determinantes do desempenho do setor de serviços é incluída, além das duas variáveis citadas acima, uma variável de PIB industrial, buscando identificar se o setor de serviços tem sua dinâmica afetada pelo setor industrial. Também são incluídas dummies referentes à macrorregião $(\mathrm{N}, \mathrm{NE}, \mathrm{CO}, \mathrm{SE}$ e $\mathrm{S})$ em que se encontra a microrregião em questão, na tentativa de controlar os efeitos da grande heterogeneidade da distribuição da atividade produtiva no Brasil (seja industrial ou de serviços).

Como a unidade de análise é territorial, entende-se que o uso de econometria espacial auxilia a identificação do modelo (ALMEIDA, 2012). Neste sentido, a busca por indicadores territoriais que possam capturar a influência exercida pela proximidade passa a ser foco da análise. Ignorar esta dependência espacial na especificação do modelo tende a violar pressupostos do modelo clássico de regressão linear, o que pode a acarretar ineficiência e viés nos estimadores, mesmo com grandes amostras.

Além da acomodação da dependência espacial no modelo, outra possibilidade de avanço metodológico está no uso do painel de dados, que além de fornecer maior número de observações permite uma maior acomodação da heterogeneidade dos dados, com possível eliminação (ou tratamento) de características particulares da unidade de análise durante a estimação.

\section{Determinantes do PIB - serviços}

A identificação do modelo dos determinantes do PIB de serviços ( $\left.\mathrm{Y}_{\text {serviços }}\right)$, usando um painel de dados espaciais, segue a estrutura abaixo:

$\mathrm{Y}_{\text {serviços it }}=\alpha+\beta_{1} *$ IDRSI $_{i t}+\beta_{2} * \mathrm{Y}_{\text {industrial it }}+\beta_{3} *$ Export $_{i t}+\beta_{4} *$
dummy2010
it

Com o PIB de serviços sendo determinado por IDRSI, PIB industrial, capacidade exportadora, e influenciado pela dinâmica econômica das regiões vizinhas (W_PIB), pela dinâmica exportadora das regiões vizinhas (W_Export) e pela dinâmica industrial das regiões vizinhas ( $\left.W_{-} Y_{\text {industrial }}\right)$. Neste caso, o termo de erro $\varepsilon_{i t}$ assume a seguinte especificação:

$\varepsilon_{\mathrm{it}}=\mu_{\mathrm{i}}+\mathrm{v}_{\mathrm{it}}$

Dentro das possibilidades do uso dos dados em painel, o modelo de efeito fixo assume que o termo de erro não observado $\left(\mu_{i}\right)$ é fixo para cada unidade de observação e o outro componente do termo de erro $\left(v_{i t}\right)$ é IID (com média 0 e variância $\sigma^{2}$ ). Para os modelos de efeitos aleatórios, o erro não observado $\mu_{i}$ é assumido aleatório e IID $\left[\mu_{i} \sim i i d\left(0, \sigma^{2}\right)\right]$, assim como o erro $v_{i t}$, sendo ainda $\operatorname{corr}\left(u_{i}, v_{i t}=0\right) \mathrm{e}$ $\operatorname{corr}\left(x_{i t}, u_{i}, v_{i t}=0\right)$.

\section{Determinantes do PIB - industrial}

Para os determinantes do PIB industrial ( $\left.Y_{\text {industrial }}\right)$, usando um painel de dados espaciais, tem-se: 
$\mathrm{Y}_{\text {industrial it }}=\alpha+\beta_{1} *$ IDRSI $_{\text {it }}+\beta_{2} *$ Export $_{\text {it }}+\beta_{3} *$ dummy2010 $_{\text {it }}+\beta_{4} *$ W_PIB $_{-}+$ $\beta_{5} * \mathrm{~W}_{-}$Export $_{\mathrm{it}}+\beta_{6} * \mathrm{~W}_{-} \mathrm{Y}_{\text {industrial it }}+\varepsilon_{\mathrm{it}}$

$\mathrm{Na}$ identificação dos determinantes da dinâmica industrial é preciso esclarecer que a variável da dinâmica industrial das regiões vizinhas ( $\left.W_{-} Y_{\text {industrial }}\right)$ utilizada no modelo não pode ser a variável observada (ALMEIDA, 2012). Caso fosse esta, existiria um viés de simultaneidade nesta determinação (a microrregião influencia seus vizinhos e também é influenciada por eles). A estratégia econométrica foi utilizar o W_Y $\mathrm{Y}_{\text {industrial }}$ estimado como instrumento para o $W_{-} Y_{\text {industrial }}$ observado, fazendo a estimação em dois estágios (em uma primeira etapa estimou-se a ponderação espacial ${ }^{5}$, para em uma segunda etapa utilizar esta variável como explicativa). Nesta especificação, a identificação do modelo assume as mesmas características descritas para o modelo anterior (determinação da dinâmica do setor de serviços), no que diz respeito ao termo de erro.

A matriz de ponderação espacial (W) utilizada nos exercícios empíricos foi a "RAINHA", que é uma matriz de proximidade geográfica de contiguidade. Isso significa que a análise econométrica levou em consideração que todos os vizinhos limítrofes às microrregiões exercem influência na dinâmica microrregional e, para tanto, foi feito o devido controle.

\section{Quadro 1}

Descrição das variáveis utilizadas na análise empírica

\begin{tabular}{|c|c|c|c|c|c|}
\hline Variáveis & Descrição & $\begin{array}{l}\text { Unidade de } \\
\text { medida }\end{array}$ & Média & Mínimo & Máximo \\
\hline \multicolumn{6}{|c|}{ Variáve is contínuas } \\
\hline IDRSI & $\begin{array}{l}\text { Índice de Desenvolvimento Regional no Sistema } \\
\text { de Inovação }\end{array}$ & Contínua & 1,14E-07 & $-0,30604$ & 15,23720 \\
\hline $\begin{array}{l}\text { PIB industrial (per } \\
\text { capta) }\end{array}$ & $\begin{array}{l}\text { Produção industrial por habitantes da } \\
\text { microrregião (a preços de 2000) - IBGE }\end{array}$ & $\mathrm{R} \$ 1.000,00$ & 1,92629 & 0,06454 & 26,42680 \\
\hline $\begin{array}{l}\text { PIB serviços (per } \\
\text { capta) }\end{array}$ & $\begin{array}{l}\text { Produção do setor de serviços por habitantes da } \\
\text { microrregião (a preços de 2000) - IBGE }\end{array}$ & $\mathrm{R} \$ 1.000,00$ & 2,83415 & 0,55378 & 21,31100 \\
\hline $\begin{array}{l}\text { Exportações (per } \\
\text { capta) }\end{array}$ & $\begin{array}{l}\text { Exportações por habitantes da microrregião } \\
\text { (FOB) - MDIC }\end{array}$ & US\$ 1.00 & 643,83 & 0 & 46989,31 \\
\hline $\begin{array}{l}\text { PIB (per capta) } \\
\text { vizinhos }\end{array}$ & $\begin{array}{l}\text { Ponderação espacial do PIB das microrregiões } \\
\text { vizinhas (a preço de 2000) - IBGE }\end{array}$ & $\mathrm{R} \$ 1.000,00$ & 5,33553 & 1,32175 & 17,05237 \\
\hline $\begin{array}{l}\text { Exportações (per } \\
\text { capta) vizinhos }\end{array}$ & $\begin{array}{l}\text { Ponderação espacial das exportações das } \\
\text { microrregiões vizinhas (FOB) - MDIC }\end{array}$ & US\$ 1.00 & 610,73 & 0 & 16354,67 \\
\hline $\begin{array}{l}\text { PIB industrial (per } \\
\text { capta) vizinhos }\end{array}$ & $\begin{array}{l}\text { Ponderação espacial do PIB industrial das } \\
\text { microrregiões vizinhas (a preço de 2000) - } \\
\text { IBGE }\end{array}$ & $\mathrm{R} \$ 1.000,00$ & 1,30652 & 0,09944 & 8,63767 \\
\hline \multicolumn{6}{|c|}{$\begin{array}{ll} & \text { Variáve is categóricas } \\
\end{array}$} \\
\hline Norte & $\begin{array}{l}\text { Dummy para microrregião localizada na região } \\
\text { norte }\end{array}$ & binária & 0,1147 & 0 & 1 \\
\hline Nordeste & $\begin{array}{l}\text { Dummy para microrregião localizada na região } \\
\text { nordeste }\end{array}$ & binária & 0,3369 & 0 & 1 \\
\hline Centro-Oeste & $\begin{array}{l}\text { Dummy para microrregião localizada na região } \\
\text { centro-oeste }\end{array}$ & binária & 0,0932 & 0 & 1 \\
\hline Sudeste & $\begin{array}{l}\text { Dummy para microrregião localizada na região } \\
\text { sudeste }\end{array}$ & binária & 0,2867 & 0 & 1 \\
\hline Sul & $\begin{array}{l}\text { Dummy para microrregião localizada na região } \\
\text { sul }\end{array}$ & binária & 0,1685 & 0 & 1 \\
\hline
\end{tabular}

O Quadro 1 descreve as variáveis utilizadas nos exercícios empíricos e o Quadro 2 apresenta a matriz de correlação das variáveis. Observa-se que a variável IDRSI, como esperado apresenta correlação positiva com as variáveis relativas ao nível de atividade

$5 \mathrm{O} W_{-} Y_{\text {industrial }}$ foi estimado seguindo a especificação: $W_{-} Y_{\text {industrial it }}=\alpha+\beta_{1} * \operatorname{IDRSI}_{i t}+\beta_{2} *$

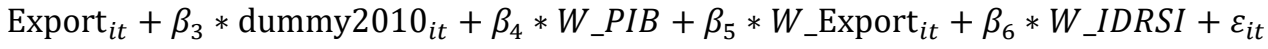


econômica. Contudo, tais correlações são relativamente baixas. Tal resultado pode ser um indicativo da já recorrentemente observada baixa conexão entre os atores do SNI brasileiro, especialmente os ligados ao ensino e à pesquisa, e a estrutura produtiva do país (ALBUQUERQUE, 1999).

Quadro 2

Matriz de correlações entre as variáveis utilizadas na análise empírica

\begin{tabular}{|c|c|c|c|c|c|c|c|c|c|}
\hline & $\begin{array}{l}\text { PIB industrial } \\
\text { (per capta) }\end{array}$ & $\begin{array}{c}\text { PIB serviços } \\
\text { (per capta) }\end{array}$ & PIB (per capta) & IDRSI & $\begin{array}{c}\text { PIB (per capta) } \\
\text { vizinhos }\end{array}$ & $\begin{array}{c}\text { Exportações } \\
\text { (per capta) }\end{array}$ & $\begin{array}{c}\text { Exportações } \\
\text { (per capta) } \\
\text { vizinhos }\end{array}$ & $\begin{array}{l}\text { PIB industrial } \\
\text { (per capta) } \\
\text { vizinhos }\end{array}$ & $\begin{array}{c}\text { PIB serviços } \\
\text { (per capta) } \\
\text { vizinhos }\end{array}$ \\
\hline $\begin{array}{l}\text { PIB industrial (per } \\
\text { capta) }\end{array}$ & 1,0000 & & & & & & & & \\
\hline $\begin{array}{l}\text { PIB serviços (per } \\
\text { capta) }\end{array}$ & 0,4998 & 1,0000 & & & & & & & \\
\hline PIB (per capta) & 0,8089 & 0,8771 & 1,0000 & & & & & & \\
\hline IDRSI & 0,1533 & 0,2634 & 0,2186 & 1,0000 & & & & & \\
\hline $\begin{array}{l}\text { PIB (per capta) } \\
\text { vizinhos }\end{array}$ & 0,4447 & 0,6618 & 0,6825 & 0,1920 & 1,0000 & & & & \\
\hline $\begin{array}{l}\text { Exportações (per } \\
\text { capta) }\end{array}$ & 0,5514 & 0,3998 & 0,5407 & 0,0664 & 0,2745 & 1,0000 & & & \\
\hline $\begin{array}{l}\text { Exportações (per } \\
\text { capta) vizinhos }\end{array}$ & 0,2782 & 0,3645 & 0,3991 & 0,1715 & 0,6024 & 0,2700 & 1,0000 & & \\
\hline $\begin{array}{l}\text { PIB industrial (per } \\
\text { capta) vizinhos }\end{array}$ & 0,4593 & 0,5357 & 0,5703 & 0,1879 & 0,8685 & 0,2444 & 0,5645 & 1,0000 & \\
\hline $\begin{array}{l}\text { PIB serviços (per } \\
\text { capta) vizinhos }\end{array}$ & 0,4196 & 0,6803 & 0,6647 & 0,2014 & 0,9533 & 0,2576 & 0,5460 & 0,7552 & 1,0000 \\
\hline
\end{tabular}

\section{Análise dos Resultados}

A análise dos resultados será feita com base nas tabelas 2 e 3 , que seguem. $\mathrm{O}$ foco da análise diz respeito a identificar a relação entre a variável IDRSI e o nível de atividade dos setores indústria e serviços para as microrregiões brasileiras. Com isso, pretende-se avaliar como os avanços na distribuição espacial recente do SNI brasileiro podem ter impactado em atividades econômicas regionais. Para tal, a Tabela 2 apresenta os resultados da estimação dos determinantes do setor de serviços enquanto a Tabela 3 apresenta os resultados da estimação dos determinantes da dinâmica industrial.

Como descrito na parte metodológica, para os dois exercícios foram feitas algumas tentativas de identificar o melhor modelo a ser estimado, representadas nas 7 especificações de MQO. Posteriormente foi feito o uso do instrumental de painel, com estimação de Efeitos Fixos e Efeitos Aleatórios. Para os dois casos foram feitos o teste de Hausman, que identificou qual é o modelo preferido.

Ainda antes de entrar na análise dos resultados dos modelos finais, cabe salientar que a primeira especificação proposta, que apenas tentava mensurar a influência exercida por IDRSI em PIB de serviços e PIB industrial, obteve o resultado previamente esperado. Com um efeito positivo e bastante significativo, indicando que o IDRSI reforça substancialmente a dinâmica destes dois setores. Em outras palavras, a presença de atores do SNI nas microrregiões geraria efeitos positivos tantos em suas atividades no setor serviços quanto em suas atividades no setor industrial. Entretanto, como já apresentado na metodologia, esta primeira especificação apresenta problemas de omissão de variáveis relevantes, enviesando os resultados.

Tabela 2

Determinantes microrregionais da dinâmica do setor de serviços (2000 e 2010) 


\begin{tabular}{|c|c|c|c|c|c|c|c|c|c|}
\hline & \multicolumn{7}{|c|}{ MQO } & \multirow{2}{*}{ Efeito fixo } & \multirow{2}{*}{ Efeito Aleatório } \\
\hline & es pecificação 1 & es pe cificação 2 & es pe cificação 3 & especificação 4 & especificação 5 & es pecificação 6 & especificação 7 & & \\
\hline \multirow{2}{*}{ cons tante } & $2.834 * * * *$ & $0.721^{\text {**** }}$ * & $0.774 * * * *$ & $0.732^{* * * *}$ & $0.620^{* * * *}$ & $0.487 * * * *$ & $0.805^{* * * *}$ & $0.347^{* * * * * *}$ & $0.619 * * *$ \\
\hline & $(0.055)$ & $(0.097)$ & $(0.091)$ & $(0.093)$ & $(0.103)$ & $(0.098)$ & $(0.218)$ & $(0.117)$ & $(0.104)$ \\
\hline IDRSI & $0.536 * * *$ & $0.253 * * * *$ & $0.261^{* * * *}$ & $0.265 * * *$ & $0.280 * * *$ & $0.283 * * *$ & $0.269^{* * * *}$ & 0.010 & 0.220 **** \\
\hline \multirow{2}{*}{$\begin{array}{c}\text { PIB industrial (per } \\
\text { capta) }\end{array}$} & & $0.242 * * * *$ & $0.157 * * *$ & 0.159 **** & $0.152 * *$ & $0.176 * * *$ & $0.172 * * *$ & -0.026 & $0.059 * *$ \\
\hline & & $(0.053)$ & $(0.059)$ & $(0.059)$ & $(0.060)$ & $(0.061)$ & $(0.060)$ & $(0.031)$ & $(0.024)$ \\
\hline \multirow{2}{*}{ Exportações (per capta) } & & & $0.001 * *$ & $0.001 * *$ & $0.001 * *$ & $0.001 * *$ & $0.001 * *$ & $0.001 * * *$ & $0.001 * * * *$ \\
\hline & & & $(0.000)$ & $(0.000)$ & $(0.000)$ & $(0.000)$ & $(0.000)$ & $(0.000)$ & $(0.000)$ \\
\hline \multicolumn{10}{|c|}{ Controle espacial } \\
\hline \multirow{2}{*}{ PIB (per capta) vizinhos } & & $0.336 * * * *$ & $0.332 * * *$ & $0.325 * * *$ & $0.362 * * *$ & $0.466 * * * *$ & $0.441 * * *$ & $0.566 * * *$ & $0.458 * * * *$ \\
\hline & & $(0.022)$ & $(0.024)$ & $(0.024)$ & $(0.029)$ & (0.038) & $(0.058)$ & (0.034) & $(0.029)$ \\
\hline \multirow{2}{*}{$\begin{array}{c}\text { Exportações (per capta) } \\
\text { vizinhos }\end{array}$} & & & & & $-0.001 * *$ & $-0.001 * *$ & $-0.001 * *$ & $-0.001 * * * *$ & $-0.001 * * *$ \\
\hline & & & & & $(0.000)$ & $(0.000)$ & $(0.000)$ & $(0.000)$ & $(0.000)$ \\
\hline \multirow{2}{*}{$\begin{array}{l}\text { PIB industrial (per } \\
\text { capta) vizinhos }\end{array}$} & & & & & & $-0.343 * * *$ & $-0.360 * * *$ & $-0.413 * * *$ & -0.331 **** \\
\hline & & & & & & $(0.098)$ & $(0.102)$ & $(0.085)$ & $(0.066)$ \\
\hline \multicolumn{10}{|c|}{ Dummy ano } \\
\hline \multirow{2}{*}{2010} & & & & $0.161 * *$ & $0.213 * * *$ & $0.179 * *$ & $0.221 * *$ & & \\
\hline & & & & $(0.078)$ & $(0.079)$ & $(0.079)$ & $(0.089)$ & & \\
\hline \multicolumn{10}{|c|}{ Dummies regionais } \\
\hline \multirow{2}{*}{ NE } & & & & & & & $-0.303^{* *}$ & & \\
\hline & & & & & & & (0.138) & & \\
\hline \multirow{2}{*}{$\mathbf{C O}$} & & & & & & & -0.234 & & \\
\hline & & & & & & & $(0.296)$ & & \\
\hline SE & & & & & & & omitida & & \\
\hline \multirow[b]{2}{*}{${ }_{1}$} & & & & & & & -0.150 & & \\
\hline & & & & & & & $(0.103)$ & & \\
\hline \multirow{2}{*}{\multicolumn{10}{|c|}{$\begin{array}{l}\text { Notas: } 1) * * *, * * \mathrm{e} * \text { representam coeficiente } \\
\text { 2) Desvio padrão robusto entre parênteses. }\end{array}$}} \\
\hline & & & & & & & & & \\
\hline \multicolumn{10}{|c|}{ 3) Matriz de ponderação espacial do tipo RAINHA } \\
\hline Fonte: Elaboração própria c & om base em IBGE & MDIC, ISI, INPI & e GeoCapes & & & & & & \\
\hline
\end{tabular}

A interpretação dos resultados da estimação dos determinantes da dinâmica do setor de serviços será feita com base nos resultados do modelo de Efeitos Fixos. O teste de Hausman ${ }^{6}$ indicou que este modelo é preferível em relação ao de efeitos aleatórios.

A primeira constatação a ser feita, com base na análise da variável de maior interesse, diz respeito ao fato de a variável IDRSI não ser estatisticamente significante, apesar de ter um efeito positivo, como se esperava a priori. Interessante notar que em todas as demais especificações esta variável teve um efeito positivo e significativo, indicando que ela reforça a dinâmica deste setor. A interpretação para a perda de significância pode estar relacionada à eliminação de características regionais (invariantes no tempo) que acabaram "extraindo" poder explicativo da variável IDRSI. Antes desta eliminação tais características estariam presentes no termo de erro e, sem este correto tratamento, o IDRSI apresentava uma significância que seria oriunda de uma variável omitida. $O$ mesmo ocorre com a variável PIB industrial, da qual seria esperado um sinal positivo, de reforço na dinâmica do setor de serviços, mas que não é estatisticamente significativo (assim como em IDRSI, em todas as demais especificações o efeito foi positivo e significativo).

Deve-se considerar que o setor serviços apresenta grande abrangência, englobando um vasto número de atividades, sendo muitas delas caracterizadas pela baixa intensidade tecnológica e restrita produtividade. Nesse sentido, acredita-se que o peso dos serviços de menor produtividade possa contribuir para fato de não ter sido alcançada significância estatística para o IDRSI no modelo acima apresentado. Ou seja, para boa parte das atividades de serviços, a presença local de atores do SNI não resultaria em melhoras diretas para a sua dinâmica. Acredita-se que uma nova especificação da variável dependente, considerando prioritariamente o produto de segmentos do setor serviços com maior grau de produtividade poderia contribuir para que os resultados fossem mais próximos ao que se pressupõe a priori quanto aos efeitos do IDRSI sobre esta atividade.

6 Os resultados do teste de Hausman, para as duas análises, estão apresentados em anexo. 
Destaca-se, ainda, que a dinâmica do setor exportador afeta positivamente o setor de serviços, mas em uma magnitude muito baixa (apesar de muito significativo, estatisticamente). Ainda em relação ao setor exportador, resultado, também, interessante se refere ao fato de a dinâmica exportadora das regiões vizinhas afetar negativamente a dinâmica dos serviços na região em análise. Isso pode significar que o setor exportador geraria maiores efeitos sobre a dinâmica econômica interna à região em que se localiza, estando entre esses resultados uma capacidade de atração de atividades de serviços localizadas em microrregiões vizinhas. Isso explicaria seus efeitos positivos sobre os serviços localizados internamente à região considerada, e os efeitos positivos gerados sobre a dinâmica dos serviços em regiões vizinhas.

O resultado da influência do PIB das regiões vizinhas sobre o setor de serviços indica sinal e significância esperados. Ou seja, a dinâmica econômica (geral) do entorno induz o setor de serviços nas microrregiões consideradas. Esse dado indica que os serviços apresentam encadeamentos regionais. Já na análise do PIB industrial, a lógica se assemelha ao setor exportador, ou seja, o dinamismo industrial das regiões vizinhas afeta negativamente o setor de serviços (dentro da microrregião). A explicação, assim como na análise do setor exportador, poderia estar relacionada à maior capacidade de geração de dinâmica do setor industrial no setor de serviços internamente à região em que este se localiza (entretanto isto não se confirmou na análise específica desta variável, que não foi estatisticamente diferente de zero).

Nos modelos em que houve a inclusão de uma dummy para identificar diferenças entre os anos de 2000 e 2010 observou-se, em todos, que o ano de 2010 apresentou um maior dinamismo no setor de serviços (coeficiente positivo e estatisticamente significante). Nesse sentido, é possível considerar que houve uma melhora no que tange à dinâmica econômica do setor serviços nas microrregiões do país ao longo do período avaliado.

A tabela 3, a seguir, apresenta os resultados da análise dos determinantes microrregionais da dinâmica do setor industrial. O teste de Hausman, para esta análise, indicou que o modelo de Efeitos Aleatórios é preferível ao modelo de efeitos fixos.

Para os determinantes da dinâmica industrial microrregional, a variável IDRSI apresenta o sinal esperado, positivo, e significativo estatisticamente, ou seja, ela atua em direção a reforçar a dinâmica industrial local. Esse resultado indica que a presença de atores do SNI influenciaria de forma positiva a atividade industrial nas microrregiões brasileiras. Trata-se de um resultado relevante, dado o processo recente de expansão do SNI brasileiro, o qual foi marcado pela busca de uma melhor distribuição espacial de seus entes. Tal resultado também é condizente com a literatura internacional, que sugere que a presença de instituições de ciência e tecnologia geograficamente próximas seria um fator indutor da dinâmica econômica, e tecnológica, no setor industrial (AUDRETSCH; FELDMAN, 1996; JAFFE, 1989). Também é possível associá-lo aos estudos realizados sobre a proximidade geográfica e a relação entre ciência e tecnologia no Brasil na indução do desenvolvimento industrial e regional (SANTOS; DINIZ, 2013; SUZIGAN; ALBUQUERQUE, 2011).

Desta forma, pode-se interpretar que a presença de atores do SNI brasileiro nas microrregiões desencadeia efeitos positivos sobre a indústria local, o que seria resultante das produções científica e tecnológica, bem como das atividades de formação e qualificação profissional inerentes à estrutura de sistemas de inovação. Sendo assim, a indústria colheria resultados positivos da proximidade junto a tais estruturas de ciência e tecnologia, sendo estes refletidos em sua maior produtividade, capacidade de inovação, e, consequentemente, maior geração de renda. Esse quadro reforça a importância do 
processo de redistribuição espacial dos entes dos SNI no país, dado que a atividade produtiva local colhe frutos de sua presença no mesmo ambiente regional.

Destaca-se que, embora, a expansão territorial da infraestrutura de ensino e pesquisa possa não resultar imediatamente na introdução de inovações de produto por empresas locais, ela pode impactar positivamente na atividade industrial. Nesse sentido, é possível mencionar os impactos positivos do ensino e da pesquisa regionalizados sobre a produtividade, uma vez que a qualificação humana tende a ampliar as capacitações produtivas locais. Além disso, a introdução de inovações de processo e incrementais, facilitadas pela existência de instituições de ensino e pesquisa podem explicar com maior clareza os seus efeitos positivos sobre a indústria regional em um intervalo de tempo relativamente curto. Outro aspecto que pode ser levado em consideração diz respeito ao aumento do investimento industrial em tais regiões, motivado justamente pela presença de uma infraestrutura local de ensino e pesquisa.

\section{Tabela 3}

Determinantes microrregionais da dinâmica industrial no brasil (2000 - 2010)

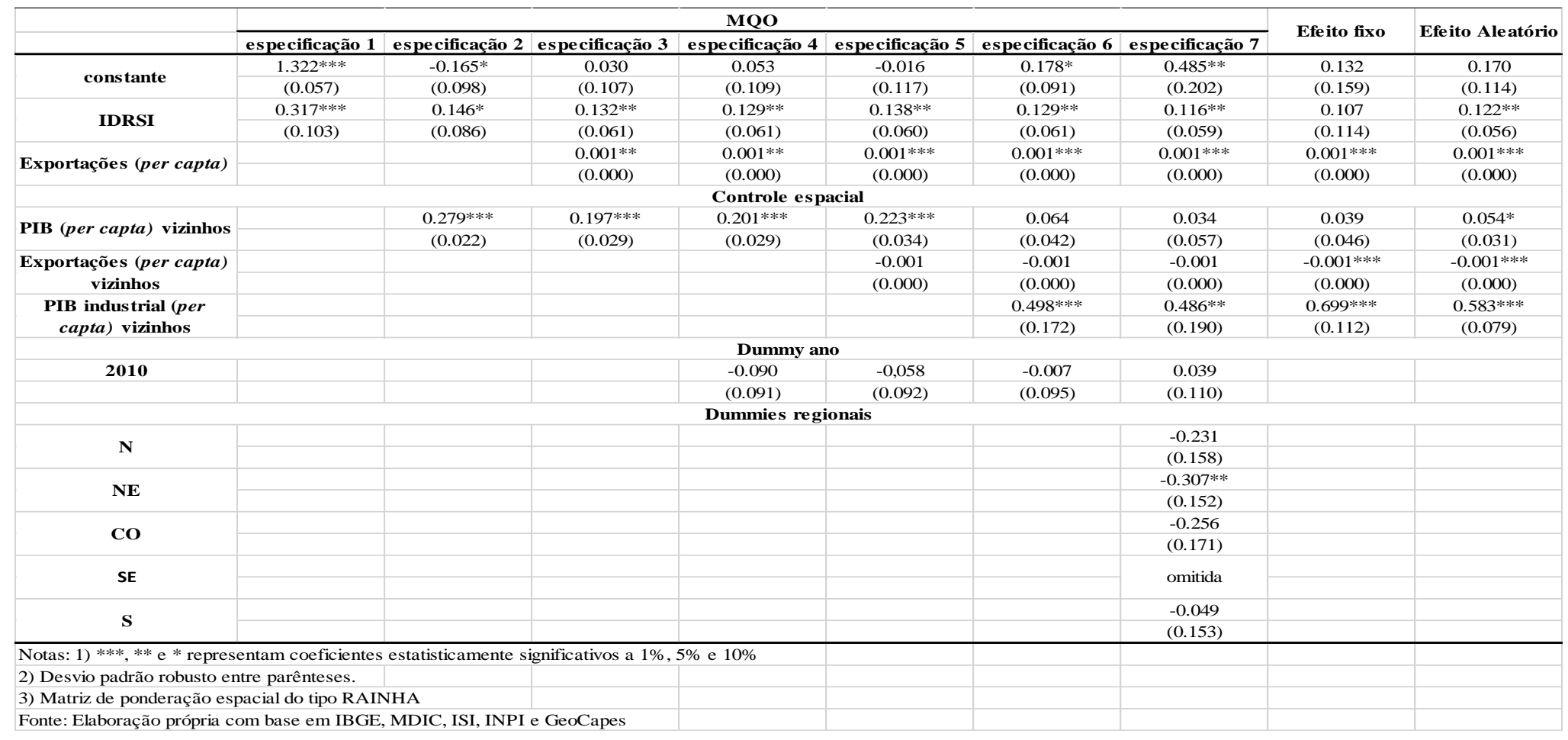

Cabe observar, no entanto, que o IDRSI não configurou o principal determinante da atividade industrial microrregional, de acordo com os dados avaliados. A variável referente à dinâmica industrial das microrregiões vizinhas foi a que apresentou o mais alto coeficiente entre as variáveis testadas no exercício empírico considerado, além de deter significância estatística. Este resultado reflete a notória tendência da indústria em se beneficiar de spillovers resultantes da localização em aglomerações produtivas (ASHEIM, 1996; COOKE, 1998). Nesse sentido, a existência de economias de aglomeração, referentes a aspectos como a infraestrutura local, a proximidade de clientes e fornecedores, a existência de oferta de trabalho qualificado e os fluxos de conhecimento proporcionados pela existência de indústrias no entorno, configuraria o principal determinante da indústria nas microrregiões observadas. 
Destaca-se que o fato de as variáveis acima mencionadas serem as com coeficientes significativos mais importantes para a dinâmica microrregional do setor industrial é condizente com as expectativas orientadas pela literatura. Além disso, tais resultados indicam os caminhos para o desenvolvimento de políticas direcionadas ao setor industrial no país, as quais devem, portanto, contemplar a abrangência territorial do SNI, assim como as aglomerações produtivas. Outro ponto a se destacar diz respeito à ordem de importância para as duas variáveis, que indicam que a dinâmica industrial do entorno teria maior impacto na dinâmica do setor do que a presença na microrregião de atores do SNI. Esse resultado pode sugerir que, perante a estrutura produtiva brasileira, as conexões e encadeamentos inerentes a uma aglomeração produtiva seriam mais relevantes para a dinâmica do setor industrial que a existência de uma estrutura local de ciência e tecnologia. Trata-se, portanto, de um resultado que pode estar ligado ao padrão estrutural da indústria brasileira, marcado pela forte presença de setores de média-baixa e baixa densidades tecnológicas. Para tais setores a perspectiva de inovação tecnológica tende a ser baixa, o que leva a menores interações para com os entes do SNI, se comparados aos setores com maior densidade tecnológica. No entanto, as relações para com clientes e fornecedores fazem com que estes setores tenham fortes conexões com outras indústrias.

Ainda sobre os resultados da estimação, tem-se que o setor exportador também afeta positivamente a dinâmica industrial, apesar de manifestar uma magnitude muito baixa de impacto. A pequena influência desta variável para a dinâmica industrial, observada no exercício empírico considerado pode estar relacionada à redução da importância do setor industrial na composição das exportações brasileiras ao longo do período considerado. Em relação a isso, deve-se considerar, ainda, a perda de competitividade da indústria de transformação brasileira no mercado internacional frente a um processo de apreciação do câmbio real (OREIRO; FEIJÓ, 2010). Com isso, é possível entender as razões pelas quais o mercado externo influenciou apenas levemente a dinâmica da indústria local, considerando o período 2000 - 2010. Ressalta-se, também, que, mantendo a ideia de que o setor exportador tenderia a reforçar apenas a dinâmica interna à região, em detrimento de afetar seu entorno, o dinamismo do setor exportador do entorno tende a afetar negativamente a dinâmica industrial interna à microrregião, como observado pela estimação de efeitos aleatórios.

O dinamismo econômico (geral) das microrregiões vizinhas também tende a afetar positivamente a dinâmica industrial interna, isso indica que a indústria se beneficia transbordamentos positivos do crescimento econômico de regiões próximas à sua localização. Logo, é perceptível que o setor industrial guarda relações com a trajetória econômica de seu entorno sob vários aspectos. Considerando o PIB per capita do entorno das microrregiões avaliadas nessa análise, é possível observar a presença de encadeamentos regionais, não diretamente ligados à indústria, que favorecem $o$ crescimento do setor numa dada localidade.

Deve-se destacar, por fim, que nas estimações de MQO, em quatro especificações em que foram incluídas as dummies de controle para diferenças entre os anos de 2000 e 2010 os resultados foram opostos à expectativa baseada na evolução para o IDRSI no período. Em todas elas os resultados obtidos mostraram não haver diferenças (estatisticamente significativas) entre os dois anos para o PIB do setor indústria. Ou seja, não houve um aumento do dinamismo do setor industrial nas microrregiões consideradas ao longo da década. Esse resultado contrasta com os dados relativos ao IDRSI, dado que esse indicador apresentou forte aumento entre os anos de 2000 e 2010. Tal ausência de mudanças na dinâmica do setor industrial, considerando as especificações do modelo em que foram inclusas dummies de ano, pode estar atrelada à 
redução do crescimento do setor na década de 2000, a qual tem sido atribuída a aspectos conjunturais como o movimento cambial do período (OREIRO; FEIJÓ, 2010). Desta forma, acredita-se que o baixo crescimento do setor possa estar relacionado à não observação de mudanças, estatisticamente significativas, na dinâmica da indústria entre os anos de 2000 e 2010, mesmo tendo o SNI brasileiro se expandido.

\section{Considerações Finais}

O presente trabalho teve como objetivo avaliar os efeitos da presença de atores do SNI sobre a atividade econômica das microrregiões brasileiras. A análise proposta foi baseada num painel de dados considerando os anos de 2000 e 2010, o qual foi utilizado com vistas a avaliar a dinâmica econômica dos setores indústria e serviços. Foi adotado um indicador, obtido por meio do método de análise fatorial, capaz de sumarizar informações relativas ao grau de desenvolvimento dos atores que compõem o SNI localizados nas microrregiões brasileiras. Nesse sentido, exercícios econométricos foram realizados para verificar os efeitos deste indicador, ou seja, da presença de atores do SNI nas microrregiões, sobre as atividades econômicas locais.

Em relação ao setor serviços, observou-se a ausência de significância estatística do IDRSI para explicar a dinâmica do setor, embora o coeficiente observado sugira uma relação positiva. Acredita-se que o fato de o setor serviços contemplar um amplo conjunto de segmentos que não se beneficiariam diretamente da presença de atores do SNI, especialmente os serviços com menor produtividade, explicaria a inexistência de relação estatística entre o IDRSI e a dinâmica do setor para o exercício aqui realizado.

Quanto à indústria, por sua vez, é estatisticamente observável a relação positiva entre a presença na microrregião de atores do SNI e a dinâmica do setor. Desta forma, é verificável a relevância da existência de uma estrutura de ciência e tecnologia local para o desempenho das atividades industriais. Tal resultado confirma estudos anteriores sobre a relevância da proximidade geográfica entre a indústria e os entes do sistema de inovação e indica a importância do processo recente de expansão do SNI brasileiro. Acredita-se que com o passar do tempo, e na medida que os relacionamentos locais entre a infraestrutura de ensino e pesquisa e o setor produtivo amadurecerem, os impactos da primeira sobre a última tendem a ser ainda maiores que os observados pro esse trabalho. Outro resultado relevante diz respeito ao peso da atividade industrial do entorno sobre o desempenho da indústria nas microrregiões avaliadas. A combinação destes resultados indica que os principais determinantes regionais da dinâmica industrial no país diriam respeito à sua localização aglomerada e à existência de uma estrutura de ciência e tecnologia local.

Destes resultados é possível concluir que a redistribuição espacial do SNI brasileiro, contemplando localidades que não contavam com seus atores, pode ser uma fonte de novas oportunidades para o desenvolvimento produtivo, sobretudo, para a indústria que se mostrou positivamente influenciável pela presença de tais estruturas. Com isso, acredita-se que as políticas públicas visando ao desenvolvimento regional devem ser associadas às políticas científica e tecnológica, considerando que a localização dos atores do SNI tem importantes repercussões para as atividades produtivas. Nesse sentido, é fundamental a continuidade do processo de expansão e redistribuição espacial do sistema brasileiro de inovação, que ainda se encontra muito abaixo dos padrões observados para os países desenvolvidos. 


\section{Referências Bibliográficas}

ALBUQUERQUE, E. National Systems of Innovation And Non-OECD Countries: Notes about a rudimentary and tentative Typology. Brazilian Journal of Political Economy, v. 19, n. 4, p. 35-54, 1999.

ALBUQUERQUE, E. et al. A Distribuição Espacial da Produção Científica e Tecnológica Brasileira : uma Descrição de Estatísticas de Produção Local de Patentes e Artigos Científicos *. RBI-Revista Brasileira de Inovação, v. 1, n. 2, p. 225-251, 2002.

ALMEIDA, E. Econometria Espacial Aplicada. Campinas: Alínea Editora, 2012.

ASHEIM, B. T. Industrial districts as "learning regions": a condition for prosperity. European Planning Studies, v. 4, n. 4, p. 7-11, 1996.

ASHEIM, B. T.; GERTLER, M. The Geography of Innovation. In: FAGERBERG, J.; MOWERY, D.; NELSON, R. R. (Eds.). . The Oxford Handbook of Innovation. Oxford: Oxford University Press, 2005. p. 291-317.

AUDRETSCH, D.; FELDMAN, M. R \& D spillovers and the geography of innovation and production. The American Ecnomic Review, v. 86, n. 3, p. 630-641, 1996.

COOKE, P. Regional innovation systems: Competitive regulation in the new Europe. Geoforum, v. 23, n. 3, p. 365-382, jan. 1992.

COOKE, P. Introduction: origins of the concept. In: BRACZYC, H.; COOKE, P.; HIDERNREICH, M. (Eds.). . Regional Innovation Systems. London: UCL Press, 1998. p. 2-25.

COOKE, P. Regional Innovation Systems, Clusters, and the Knowledge Economy. Industrial and Corporate Change, v. 10, n. 4, p. 44-45, 2001.

DOLOREUX, D. What we should know about regional systems of innovation. Technology in Society, v. 24, n. 2, p. 243-263, 2002.

EDQUIST, C. Systems of Innovation: perspectives and challenges. In: FARCEBERG, J.; MOWERY, D.; NELSON, R. R. (Eds.). . The Oxford Handbook of Innovation. Oxford: Oxford University Press, 2005. p. 181-208.

FLORIDA, R. Toward the Learning Region. Futures, v. 27, n. 5, p. 527-536, 1995.

FREEMAN, C. Technology Policy and Economic Performance. London: Pinter Publishers, 1987.

FREEMAN, C. The "National System of Innovation"in historical perspective. Cambridge Journal of economics, v. 19, p. 5-24, 1995.

FREEMAN, C. Continental, national and sub-national innovation systems complementarity and economic growth. Research Policy, v. 31, p. 191-211, 2002.

GRILICHES, Z. Patent statistics as economic indicator: A survey. Journal of Economic Literature, v. 28, n. 3301, p. 1324-1330, 1990.

ISAKSEN, A. Building Regional Innovation Systems: Is Endogenous Industrial Development Possible in the Global Economy? Globalisation: A Challenge for Local Industrial Policy Regionalisation as an Aspect of Economic Globalisation. Canadian Journal of Regional Science, v. 24, n. 1, p. 101-120, 2001.

JAFFE, A. Real Effects Of Academic Research. American Economic Review, v. 79, n. 
5, p. 957-971, 1989.

JOHNSON, B. Institutional Learning. In: LUNDVALL, B.-A. (Ed.). . National Systems of Innovation: towards a theory of innovation and interactive learning. London: Pinter Publishers, 1995. p. 23-44.

LUNDVALL, B.-A. Introduction. In: LUNDVALL, B.-A. (Ed.). . National Systems of Innovation: towards a theory of innovation and interactive learning. 2. ed. London: Pinter Publishers, 1995. p. 1-19.

MINGOTI, S. Análise de Dados Através de Métodos de Estatística Multivariada: uma abordagem aplicada. Belo Horizonte: Editora UFMG, 2005.

NELSON, R. R.; ROSENBERG, N. Technical Innovation and National Systems. In: NELSON, R. (Ed.). . National Innovation Systems. New York, Oxford: Oxford University Press, 1993. p. 3-21.

OINAS, P.; MALECKI, E. Spatial Innovation Systems. In: MALECKI, E.; OINAS, P. (Eds.). . Making Connections: Technological Learning and Regional Economic Change. Aldershot: Ashgate, 1999.

OREIRO, J. L.; FEIJÓ, C. A. Desindustrialização: conceituação, causas, efeitos e o caso brasileiro. Revista de Economia Política, v. 30, n. 2, p. 219-232, 2010.

RIBEIRO, L. et al. Matrices of science and technology interactions and patterns of structured growth: implications for development. Scintometrics, Textos para Discussão. v. 8, p. 55-75, 2010.

SANTOS, U. P. DOS. A dimensão espacial da sistema nacional de inovação e seus impactos regionais na economia brasileira. Belo Horizonte: Tese de doutoramento, 2014.

SANTOS, U. P. DOS; DINIZ, C. C. A interação universidade-empresa na siderurgia de Minas Gerais. Nova Economia, v. 23, n. 2, p. 279-306, 2013.

SUZIGAN, W.; ALBUQUERQUE, E. The underestimated role of universities for the Brazilian system of innovation. Revista de Economia Política, v. 31, n. 1, p. 3-30, 2011.

\section{Anexos}

Teste de Hausman para a estimação dos determinantes da dinâmica do setor de serviços

\begin{tabular}{|c|c|c|c|c|c|}
\hline \multirow{2}{*}{\multicolumn{2}{|c|}{$\begin{array}{l}\text { (b) } \\
\mathrm{fe}\end{array}$}} & \multicolumn{2}{|l|}{ (B) } & \multicolumn{2}{|c|}{$\operatorname{sqrt}\left(\operatorname{diag}\left(\mathrm{V} \_b-\mathrm{V} \_\mathrm{B}\right)\right)$} \\
\hline & & re & Difference & S.E. & \\
\hline index $\mid$ & .010 & & .1993875 & -.1891853 & .0685476 \\
\hline ind_ppc & -.02 & 3536 & .0669533 & -.0933069 & .0196258 \\
\hline w_ppc & .56 & 5329 & .5240068 & .0415261 & .0218085 \\
\hline $\mathrm{xppc}$ & .000 & 793 & .0001571 & .0000221 & $6.02 \mathrm{e}-06$ \\
\hline w_xppc & & 1172 & -.0001101 & $-7.11 \mathrm{e}-06$ & .000017 \\
\hline w_ind_pp & & 25378 & -.3980217 & -.0145161 & .0539629 \\
\hline
\end{tabular}


$\mathrm{b}=$ consistent under Ho and Ha; obtained from xtreg

$\mathrm{B}=$ inconsistent under Ha, efficient under Ho; obtained from xtreg

Test: Ho: difference in coefficients not systematic

$$
\begin{gathered}
\operatorname{chi} 2(5)=(b-B)^{\prime}\left[\left(V_{-} b-V \_B\right)^{\wedge}(-1)\right](b-B) \\
=\quad 37.37 \\
\text { Prob }>\text { chi } 2=0.0000
\end{gathered}
$$

\begin{tabular}{|c|c|c|c|c|}
\hline$\quad(b$ & (B) & (b-B) & \multicolumn{2}{|c|}{ sqrt(diag(V_b-V_B $))$} \\
\hline index | & .107497 & .1220654 & -.0145684 & .0990662 \\
\hline w_ppc | & .0386095 & .0538391 & -.0152296 & .0331996 \\
\hline xppc| & .0002677 & .0003022 & -.0000345 & $6.88 \mathrm{e}-06$ \\
\hline w_xppc & -.000166 & -.0001509 & -.0000151 & .0000257 \\
\hline w_ind_ppc | & | . .6988473 & $3 \quad .5832819$ & .1155654 & .0791036 \\
\hline
\end{tabular}

Teste de Hausman para a estimação dos determinantes da dinâmica do setor industrial ---- Coefficients ----

Test: Ho: difference in coefficients not systematic

$$
\begin{gathered}
\operatorname{chi} 2(4)=(b-B)^{\prime}\left[\left(V_{-} b-V \_B\right)^{\wedge}(-1)\right](b-B) \\
=\quad 4.26 \\
\text { Prob }>\text { chi2 }=\quad 0.3715
\end{gathered}
$$

